\title{
Review and recent results on Stewart Gough platforms with self-motions
}

\author{
Georg Nawratil \\ Institute of Discrete Mathematics and Geometry, Vienna University of Technology, \\ Wiedner Hauptstrasse 8-10/104, Vienna, A-1040, Austria \\ nawratil@geometrie.tuwien.ac.at
}

Keywords: Self-motion, Stewart Gough platform, Borel Bricard problem

\begin{abstract}
In Section 1, we give a review on Stewart Gough (SG) platforms with self-motions and the related Borel Bricard problem. Moreover, in Section 2, we report about recent results achieved by the author on this topic (SG platforms with type II DM self-motions). In context of these results, we also present two new theorems in Section 3, which open the way for addressed future work. In Section 4, we give some final remarks on planar SG platforms with type I DM self-motions and formulate a central conjecture.
\end{abstract}

\section{Introduction}

A Stewart Gough (SG) platform is a parallel manipulator consisting of a moving platform, which is connected via six sphercial-prismatic-universal legs with the base, where the spherical and universal joints are passive and the prismatic joints are active. Therefore, the geometry of a SG platform is given by the six base anchor points $M_{i}$ and by the six platform anchor points $m_{i}$ for $i=1, \ldots, 6$. A $\mathrm{SG}$ platform $m_{1}, \ldots, M_{6}$ is called planar if $m_{1}, \ldots, m_{6}$ as well as $M_{1}, \ldots, M_{6}$ are coplanar.

If the geometry of the SG platform and the six leg lengths are given, then the manipulator is in general rigid in one of its 40 possible assembly modes. But, under particular conditions the manipulator can have $n>0$ degrees of freedom. The resulting $n$-parametric motion is called selfmotion. Clearly, in each pose of a self-motion the SG platform is singular; i.e. the carrier lines of the prismatic legs belong at least to a linear line complex (cf. [1]).

1.1 Historical background. All self-motions of SG manipulators are solutions to the still unsolved problem posed by the French Academy of Science for the Prix Vaillant of the year 1904, which reads as follows:"Determine and study all displacements of a rigid body in which distinct points of the body move on spherical paths."

The papers of Borel [2] and Bricard [3] were awarded prizes, but both authors only presented partial solutions as they assumed that there are linear relations between the direction cosines of the motion (for more details see [4,5]). Therefore this problem is also known as Borel Bricard (BB) problem. Moreover, displacements where distinct points have spherical paths are also called BB motions.

But, it should be noted that some results on BB motions were already known before the year 1904:

(a) Chasles [6] recognized within a static context, that if the points of two conic sections are in a projective correspondence, then there exists a spatial motion, which keeps the corresponding points at fixed distance.

(b) The only non-trivial motion, where all points of the moving system have spherical paths, was presented $^{1}$ by Bricard [7] in 1896.

(c) Moreover, in 1897 Bricard [8] proved the existence of three types of flexible octahedra in the Euclidean 3-space.

(d) In 1898, Duporcq [9] stated his famous theorem (for more details see [10]).

\footnotetext{
${ }^{1}$ The uniqueness of this motion was later proven by Bricard in [3].
} 
(e) The motions where all points of a straight line have spherical trajectories where studied by Duporcq [11], Mannheim [12] and by Darboux in his note to the book of Kœnigs [13].

It was not until 1937 that a further important contribution to the solution of the BB problem was given by Krames study [14,15] of line-symmetric motions (cf. [16]). All later investigations of BB motions were done in the context of self-motions of SG platforms, which are reviewed next.

1.2 Stewart Gough platforms with self-motions. It is well known, that manipulators, which are singular in every possible configuration, possess self-motions in each pose because they are redundant. These SG platforms are called architecturally singular [17] and their designs are characterized in $[18,19,20,21]$ for the planar case and in [22,23] for the non-planar one. ${ }^{2}$ Therefore, we are only interested in non-architecturally singular SG platforms with self-motions. Until now only few examples of this type are known, as their computation is a very complicated task:

The first paper mentioning self-motions, was written by Husty and Zsombor-Murray [28], where SG platforms with Schönflies self-motion of item (b) ${ }^{3}$ were reported. Beside these manipulators, further SG platforms with a line-symmetric self-motion (which was already known to Borel [2], Bricard [3] and Krames [15]) was given in Zsombor-Murray et al. [30].

Moreover, Husty and Karger [31] proved that the list of Schönflies Borel Bricard motions given by Borel [2] is complete.

Karger studied also planar parallel manipulators of SG type with affinely equivalent platform and base [32] (for the special cases of equiform and congruent platforms see [33] and [34], respectively) with respect to their self-motions.

The self-motions of TSSM designs (and 6-3 SG platforms, respectively) with intersecting rotary axes correspond to Bricard's flexible octahedra [8]. Recently, a complete list of self-motions of TSSM designs (and 6-3 SG platforms, respectively) was given by the author in [35,36] as byproduct of the determination of all flexible octahedra in the projective extension of the Euclidean 3space [37]. Moreover, the author presented in Theorem 8 of [38] a result on the self-motional behaviour of a remarkable set of planar Schönflies-singular SG platforms.

The classification of all self-motions of the original SG platform was done by Karger and Husty [39]. Based on this study, Karger [40,41] presented a method for designing planar SG platforms with self-motions of the type $e_{0}=0$, where $e_{0}$ denotes an Euler parameter.

Further SG platforms, where the self-motions have a planar spherical image, can be computed with a pure algebraic method presented by Geiß and Schreyer [42]. This publication also shows the limitation of the proposed method and that geometric-kinematic considerations are essential for the solution of the BB problem.

a)

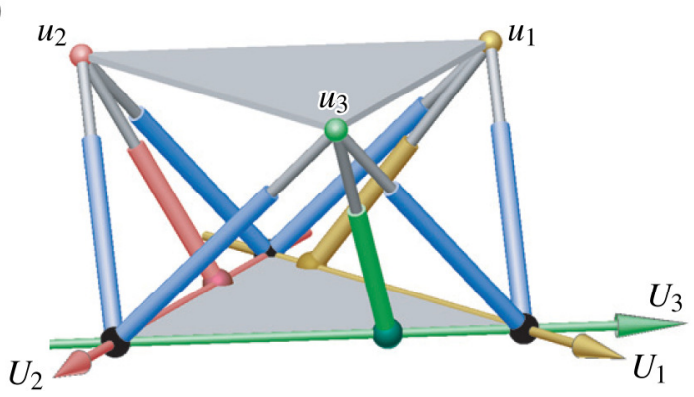

b)

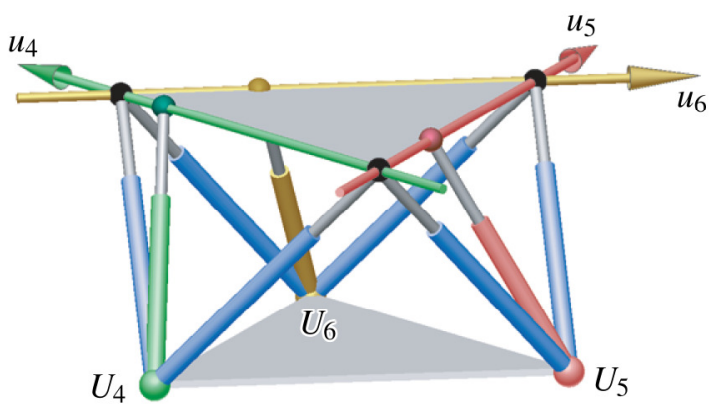

Figure 1. Octahedral manipulator: (a) Cubic $\mathrm{C}$ of base anchor points. (b) Cubic $\mathrm{c}$ of platform anchor points. Moreover, $u_{i}$ and $U_{i}(i=1, \ldots, 6)$ are displayed as well as some additional legs of $\Lambda$.

\footnotetext{
${ }^{2}$ Nevertheless, architecturally singular designs are well studied, less is known about their self-motions (cf. [24,25,26]). As demonstrated by Sommese and Wampler (e.g. [27]) the method of numerical continuation is a powerful tool for the numeric computation of these self-motions.

${ }^{3}$ Planar SG platforms of this type are also called polygon platforms (cf. [29]).
} 


\section{Recent results on SG platforms with self-motions}

In recent publications $[43,44,45,46]$ of the author, it was shown, that based on the geometrickinematic approach of redundancy, amazing new results can be achieved in the field of self-motions of SG platforms. In this section we give a short overview of these results.

Mielczarek et al. [47] investigated redundant SG platforms with a maximal forward kinematics solution set. They showed that the set $\Lambda$ of additional legs, which can be attached to a given planar SG platform $m_{1}, \ldots, M_{6}$ without restricting the forward kinematics, is determined by a linear system of equations given in Eq. (30) of [47]. As the solvability condition of this system is equivalent with the criterion given in Eq. (12) of [48], also the singularity surface of the manipulator does not change by adding legs of $\Lambda$. Moreover, it was shown in [47], that in the general case $\Lambda$ is oneparametric and that the base anchor points as well as the corresponding platform anchor points of $\Lambda$ are located on planar cubic curves $\mathrm{C}$ and $\mathrm{c}$ (see Fig. 1). Note that $\mathrm{C}$ and $\mathrm{c}$ have to do with the cubic correspondence of point paths and the centers of their osculating spheres (cf. page 142 of [49]).

Assumption 1. We assume that there exist such cubics $c$ and $C$ (which can also be reducible) in the Euclidean domain of the platform and the base, respectively.

The correspondence between the points of $\mathrm{C}$ and $\mathrm{c}$ in the complex projective extension $P^{3}$ of the Euclidean 3-space is determined by the geometry of the manipulator $m_{1}, \ldots, M_{6}$ and can be computed according to $[47,48]$. As this correspondence has not to be a bijection (see Fig. 1), a point $\in P^{3}$ of $\mathrm{c}$ or $\mathrm{C}$, respectively, is in general mapped to a non-empty set of points $\in P^{3}$ of $\mathrm{C}$ or $\mathrm{c}$, respectively. We denote this set by the term corresponding location and indicate this fact by the usage of brackets \{\} . In $P^{3}$ the cubic $\mathrm{C}$ has three ideal points $U_{1}, U_{2}, U_{3}$ and we can compute the corresponding locations $\left\{u_{1}\right\},\left\{u_{2}\right\},\left\{u_{3}\right\}$ of c. We denote the ideal points of $\mathrm{c}$ by $u_{4}, u_{5}, u_{6}$ and compute the corresponding locations $\left\{U_{4}\right\},\left\{U_{5}\right\},\left\{U_{6}\right\}$ of C (see also Fig. 1).

Assumption 2. For guaranteeing a general case, we assume that each of the corresponding locations $\left\{u_{1}\right\},\left\{u_{2}\right\},\left\{u_{3}\right\},\left\{U_{4}\right\},\left\{U_{5}\right\},\left\{U_{6}\right\}$ consists of a single point. Moreover, we assume that no four collinear platform anchor points $u_{i}$ or base anchor points $U_{i}(i=1, \ldots, 6)$ exist.

Now the basic idea can simply be expressed by attaching the special legs $\overline{u_{i} U_{i}}$ of $\Lambda$ with $i=1, \ldots, 6$ to the manipulator $m_{1}, \ldots, M_{6}$. The attachment of the leg $\overline{u_{i} U_{i}}$ for $i \in\{1,2,3\}$ corresponds with the so-called Darboux constraint (cf. [50] and Darboux's note to the book of Kœnigs [13]), that the $u_{i}$ moves in a plane of the fixed system orthogonal to the direction of the ideal point $U_{i}$ (see Fig. 2a). The attachment of the leg $\overline{u_{i} U_{i}}$ for $i \in\{4,5,6\}$ corresponds with the so-called Mannheim constraint (cf. [12,51]), that a plane of the moving system orthogonal to $u_{i}$ slides through $U_{i}$ (see Fig. 2b).

a)

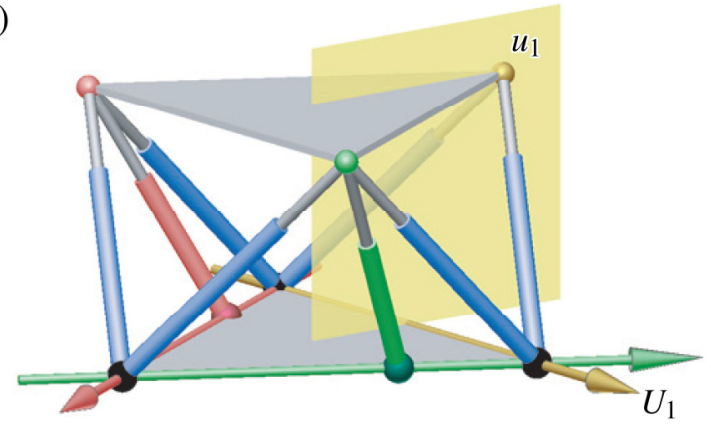

b)

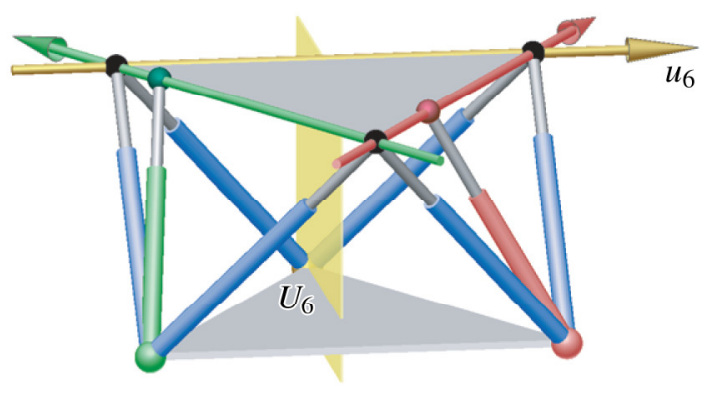

Figure 2. Octahedral manipulator: (a) Darboux constraint $(i=1)$. (b) Mannheim constraint $(i=6)$. 
By removing the originally six legs $\overline{m_{i} M_{i}}$ with $i=1, \ldots, 6$ we remain with the manipulator $u_{1}, \ldots, U_{6}$, which is uniquely determined due to Assumption 1 and 2. Moreover, under consideration of these two assumptions, the following statement holds (cf. [43]):

Theorem 1. The manipulator $u_{1}, \ldots, U_{6}$ is redundant and therefore architecturally singular. Moreover, all anchor points of the platform $u_{1}, \ldots, u_{6}$ and of the base $U_{1}, \ldots, U_{6}$ are distinct.

It was also proven in [43] that there only exist type I and type II Darboux Mannheim (DM) selfmotions, where the definition of types reads as follows:

Definition 1. Assume $\Psi$ is a one-parametric self-motion of a non-architecturally singular $S G$ platform $m_{1}, \ldots, M_{6}$. Then $\Psi$ is of type $n D M$ if the corresponding architecturally singular manipulator $u_{1}, \ldots, U_{6}$ has a $n$-parametric self-motion.

In $[44,45]$ the author proved the necessity of three conditions for obtaining a type II DM selfmotion. Moreover, a geometric interpretation of these conditions was given, which also identifies a new property of type 1 Bricard octahedra (see Fig. 3). Based on these necessary conditions, the author [46] was able to determine all planar SG platforms (fulfilling Assumption 1 and 2) with a type II DM self-motion. These manipulators are either generalizations of type 1 Bricard octahedra (12-dimensional solution set) or special polygon platforms (cf. footnote 3; 11-dimensional solution set). Moreover, it was shown that the type II DM self-motions of all these SG platforms are linesymmetric and octahedral, where the latter property is defined as follows:

Definition 2. A DM self-motion is called octahedral ${ }^{4}$, if the following triples of points are collinear for $i \neq j \neq k \neq i$ and $i, j, k \in\{1,2,3\}$ :

$$
\left(u_{i}, u_{j}, u_{6}\right),\left(u_{i}, u_{k}, u_{5}\right),\left(u_{j}, u_{k}, u_{4}\right),\left(U_{4}, U_{5}, U_{k}\right),\left(U_{5}, U_{6}, U_{i}\right),\left(U_{4}, U_{6}, U_{j}\right) .
$$

a)

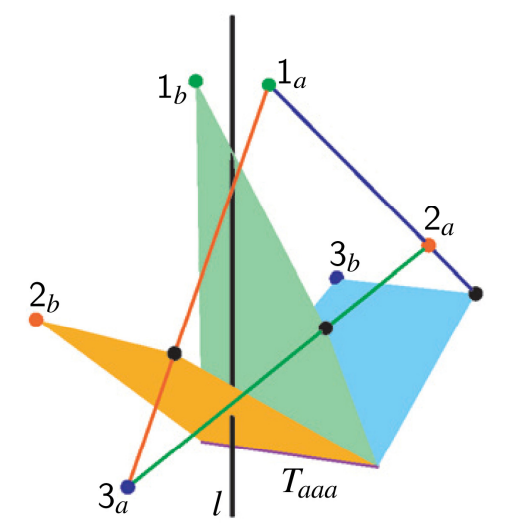

b)

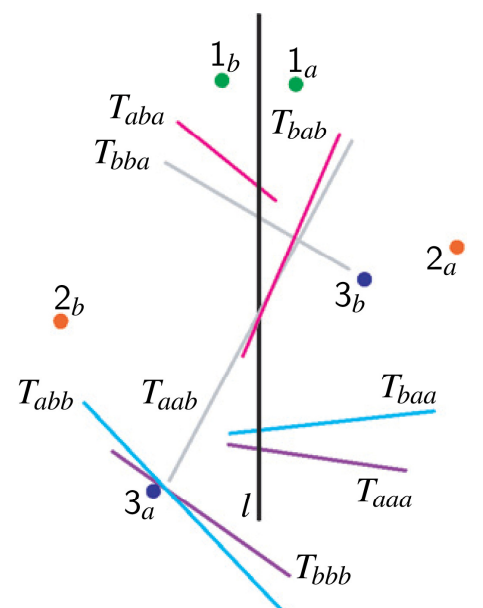

Figure 3. The vertices $v_{a}$ and $v_{b}$ of a type 1 Bricard octahedron $1_{a}, 1_{b}, 2_{a}, 2_{b}, 3_{a}, 3_{b}$ are symmetric with respect to the line $l$ for $v \in\{1,2,3\}$. The following three planes have a common line $T_{i j k}$ : (i) plane orthogonal to $\left[1_{i}, 2_{j}\right]$ though $3_{k^{\prime}}$, (ii) plane orthogonal to $\left[2_{j}, 3_{k}\right]$ though $1_{i^{\prime}}$, (iii) plane orthogonal to $\left[3_{k}, 1_{i}\right]$ though $2_{j^{\prime}}$, with $i \neq i^{\prime}, j \neq j^{\prime}, k \neq k^{\prime}$ and $i, j, k, i^{\prime}, j^{\prime}, k^{\prime} \in\{a, b\}$. This is illustrated for $i=j=k=a$ in (a). In (b) all eight possible axes $T_{i j k}$ are displayed.

\footnotetext{
${ }^{4}$ The reason for this nomenclature, which was introduced by the author in [43], is that all octahedral manipulators have this point-configuration. For $\mathrm{i}=1, \mathrm{j}=2$ and $\mathrm{k}=3$ this can be seen in Fig. 1.
} 


\section{New results in the context of Assumption 1 and 2}

In the following we present the basic result for the determination of all planar SG platforms with a type II DM self-motion, where Assumption 1 holds in contrast to Assumption 2. For the study of this case, which is dedicated to future research, we can always assume without loss of generality (w.l.o.g.) that four platform anchor points $u_{i}$ or four base anchor points $U_{i}$ are collinear, due to the following theorem:

Theorem 2. Given is a planar SG platform fulfilling Assumption 1. If one cannot choose anchor points within $\left\{u_{1}\right\},\left\{u_{2}\right\},\left\{u_{3}\right\},\left\{U_{4}\right\},\left\{U_{5}\right\},\left\{U_{6}\right\}$ such that four platform anchor points $u_{i}$ or base anchor points $U_{i}$ are collinear, then each of these corresponding locations has to consist of a single point.

Proof: We choose an arbitrary manipulator $u_{1}, \ldots, U_{6}$ within the set of all possible manipulators determined by the corresponding locations $\left\{u_{1}\right\},\left\{u_{2}\right\},\left\{u_{3}\right\},\left\{U_{4}\right\},\left\{U_{5}\right\},\left\{U_{6}\right\}$. For the same reasons as given in the proof of Theorem 1 of [43], this manipulator is redundant and therefore architecturally singular. Moreover, due to $[18,20]$ we know that architecture shakiness of a planar manipulator does not change under even different projectivities of the platform and the base. This property is important for the following argumentation:

Due to Lemma 3 of [18], there always exist four pairs of anchor points such that the anchor points in the platform and the corresponding ones in the base form quadrilaterals. After permutation of indices, we can assume w.l.o.g. that these four pairs equal $\left(u_{i}, U_{i}\right)$ for $i=1, \ldots, 4$. Then we apply projectivities to the platform and the base such that the anchor points have the following projective coordinates in the platform and the base, respectively:

$$
u_{1}=U_{1}=(1: 0: 0), u_{2}=U_{2}=(0: 1: 0), u_{3}=U_{3}=(0: 0: 1), u_{4}=U_{4}=(1: 1: 1) \text {. }
$$

Now we have exactly the same situation as in Section 3 of [20], where Röschel and Mick considered the regular projectivity $\kappa$ determined by $u_{i} \mapsto U_{i}$ for $i=1, \ldots, 4$. They demonstrated that there only exist more than six pairs of anchor points if $U_{5}=u_{5} \kappa$ holds. In this case all corresponding point pairs on the conic sections $k$ and $k \kappa$ determined by $u_{1}, \ldots, u_{5}$ and $U_{1}, \ldots, U_{5}$, respectively, are possible anchor points. Note that also $U_{6}=u_{6} \kappa$ holds and that this case corresponds with item (a) of Section 1.1. As any regular projectivity $\kappa$ is a bijection, each of the corresponding locations has to consist of single point, which finishes the proof.

Under consideration that $\Gamma$ denotes the set of planar architecturally singular manipulators with no four points collinear, which do not belong to item (a) of Section 1.1, we formulate the following theorem:

Theorem 3. To any planar SG platform $m_{1}, \ldots, M_{6}$ with exception of the set $\Gamma$, at least a oneparametric set of legs $\Lambda$ can be attached without changing the forward kinematics and singularity surface.

Proof: For the proof we consider the linear system of equations given in Eq. (30) of [47] (cf. second paragraph of Section 2):

$\mathbf{D}\left(\lambda_{1}, \ldots, \lambda_{6}\right)^{T}=\delta_{7}^{T}$ with $\mathbf{D}=\left(\delta_{1}^{T}, \ldots, \delta_{6}^{T}\right)$ and $\delta_{i}=\left(1, a_{i}, b_{i}, A_{i}, B_{i}, a_{i} A_{i}, a_{i} B_{i}, b_{i} A_{i}, b_{i} B_{i}\right)$ 
where $\left(a_{i}, b_{i}\right)$ and $\left(A_{i}, B_{i}\right)$ are the coordinates of the platform and base points, respectively. Moreover, the points with index 7 represent the anchor points of the additional legs of $\Lambda$. In the following, we have to distinguish two cases:

- For $\operatorname{rank}(\mathbf{D})<6$ the manipulator $m_{1}, \ldots, M_{6}$ is architecturally singular (cf. Remark 1 of [20]). Due to the considerations given in the proof of Theorem 2, we only have to investigate the 12 cases of architecturally singular SG platforms with four points on a line, which were listed by Karger in Theorem 3 of [22]. Due to the geometric interpretation given in Section 5.1 of [22] for the cases 1-10 and in Section 3 of [19] for the cases 11-12, it is clear that we can attach in each case at least a one-parametric set of legs without changing the forward kinematics and singularity surface.

- If the rank of $\mathbf{D}$ is maximal, i.e. $\operatorname{rank}(\mathbf{D})=6$, then we can solve six from the nine equations of Eq. (3) for $\lambda_{1}, \ldots, \lambda_{6}$. Plugging the resulting expressions in the remaining equations yield three conditions in four unknowns $\left(a_{7}, b_{7}, A_{7}, B_{7}\right)$. Therefore, we get at least a one-parametric solution set, which finishes the proof.

Beside the result of Theorem 3, only the following non-trivial exceptional case of Assumption 1 is known to the author ${ }^{5}$ : To a planar SG platform, where the platform and base anchor points are related by a projectivity (so-called planar projective SG platform), one can add a two-parametric set of legs without changing the direct kinematics and singularity surface, whereas the correspondence of anchor points is given by the projectivity itself (cf. [52]). In this context it should be noted, that based on this redundancy property, the author [52] was recently able to prove the following result:

Theorem 4. Non-architecturally singular planar projective SG platforms can only have elliptic self-motions (cf. Definition 1 of [52]) or pure translational self-motions. In the latter case the projectivity has to be a regular affinity $\boldsymbol{t}+\boldsymbol{T} \boldsymbol{x}$ ( $\Rightarrow$ planar affine SG platform), where the singular values $s_{1}$ and $s_{2}$ of the $2 \times 2$ transformation matrix $\boldsymbol{T}$ with $0<s_{1} \leq s_{2}$ fulfill $s_{1} \leq 1 \leq s_{2}$.

For more details on these self-motions ${ }^{6}$ and further properties of planar projective (affine) SG platforms, we refer to [52]. But, it should be mentioned, that all planar affine SG platforms, with exception of those given in Theorem 4, only have "ordinary" singularities causing a local shakiness of the manipulator but no self-motion itself. Note that a self-motion is extremely dangerous in practice, as the manipulator collapse under the force of gravity in an uncontrollable manner, which can damage or even destroy the manipulator and endangers the operators. Therefore such classes of manipulators, which are free of self-motions, are also of interest for designers.

a)

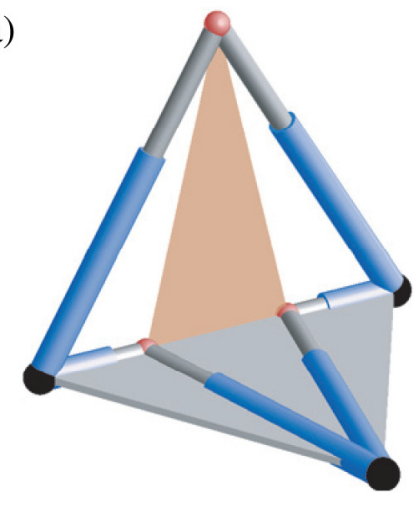

b)

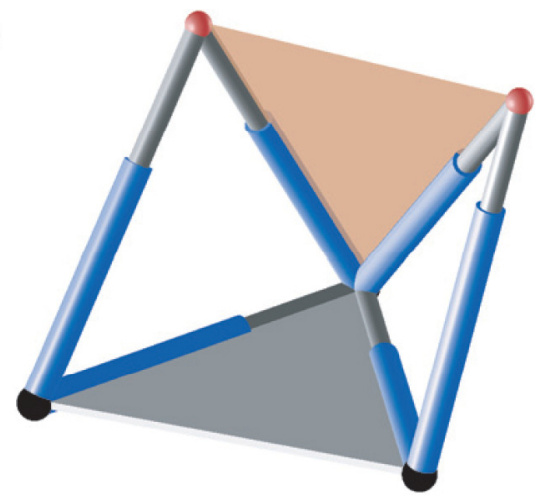

Figure 4. Octahedral manipulator: (a) Butterfly motion. (b) Spherical four-bar motion.

\footnotetext{
${ }^{5}$ A completion of this list of special cases is dedicated to future research.

${ }^{6}$ Until now it is open whether planar projective SG platforms with elliptic self-motions even exist, as no example is known to the author so far. The study of this problem is also dedicated to future research.
} 


\section{Final remarks and central conjecture}

Finally, we should also devote some words to type I DM self-motions under consideration of the following definition, which refines the proposed classification scheme (cf. Definition 1 and 2) of SG platforms with self-motions:

Definition 3. Assume a planar SG platform is given, where one can add the set 1 of legs ${ }^{7}$ without changing the direct kinematics and singularity surface. A self-motion of this manipulator is called degenerated, if one can add further legs beside $\Lambda$ to the planar manipulator without restricting the self-motion.

Until now the following type I DM self-motions are known to the author, which can be classified as follows:

i. The butterfly motion (see Fig. 4a) and the spherical four-bar motion (see Fig. 4b) of the octahedral manipulator [53] and the corresponding motions of the original SG platform [39]. Note that all these self-motions are degenerated and that they are pure spherical motions. Moreover, it can easily be seen that any SG platform with four collinear anchor points possesses a butterfly self-motion.

ii. In general ${ }^{8}$, a polygon platform (cf. footnote 3 ) possesses a degenerated type I DM selfmotion, which is not a pure spherical motion but a Schönflies motion.

iii.Non-degenerated type I DM self-motions are the self-motions of Bricard octahedra of type 2 and type 3 (cf. [8]).

In view of all SG platforms with self-motions, known to the author (cf. Section 1, 2, 3 and 4), we have good reasons to close this paper with the following central conjecture:

Conjecture 1. All non-degenerated one-parametric self-motions of non-architecturally singular planar SG platforms, fulfilling Assumption 1 and 2, are octahedral.

\section{Summary}

In Section 1 we reviewed literature with respect to SG platforms with self-motions and the related historical background in form of the Borel Bricard problem. To the best knowledge of the author the presented list of relevant references given in the paper is as complete as possible.

Moreover, in Section 2 we reported about recent results on SG platforms with type II DM selfmotions, which were achieved by the author within the last year.

In Section 3, we presented two new theorems, which open the way for addressed future work on SG platforms with self-motions. In this context, we also described a set of SG platforms which is free of self-motions.

Moreover, in Section 4 we listed all planar SG platforms with type I DM self-motions known to the author. Finally, we closed the paper by a central conjecture, which has to be proved or disproved in the future.

\section{Acknowledgements}

This research is supported by Grant No. I 408-N13 of the Austrian Science Fund FWF within the project "The Study of Structure and Properties of Flexible Polyhedra and Frameworks in Euclidean and Non-Euclidean Spaces of Dimension 3 and Higher", an international cooperation between FWF and RFBR, the Russian Foundation for Basic Research.

\footnotetext{
${ }^{7}$ Note that $\Lambda$ can also be empty $\leftrightarrow$ planar SG platform belongs to the set $\Gamma$.

${ }^{8}$ Only for the special polygon platforms mentioned in the paragraph before Definition 2, we get degenerated type II DM self-motions, which are also Schönflies motions.
} 


\section{References}

[1] J.-P. Merlet, Singular Configurations of Parallel Manipulators and Grassmann Geometry, J. Robot. Res. 8:5 (1992) 45-56.

[2] E. Borel, Mémoire sur les déplacements à trajectoires sphériques, Mém. présenteés par divers savants, Paris 2:33 (1906) 1-128.

[3] R. Bricard, Mémoire sur les déplacements à trajectoires sphériques, J. École Polyt. 2:11 (1906) $1-96$.

[4] M. Husty, E. Borel's and R. Bricard's Papers on Displacements with Spherical Paths and their Relevance to Self-Motions of Parallel Manipulators, in: M. Ceccarelli (Ed.), International Symposium on History of Machines and Mechanisms, Kluwer, 2000, pp. 163-172.

[5] H. Vogler, Bemerkungen zu einem Satz von W. Blaschke und zur Methode von Borel-Bricard, Grazer Math. Ber. 352 (2008) 1-16.

[6] M. Chasles, Sur les six droites qui peuvent étre les directions de six forces en équilibre, Comptes Rendus des Séances de l'Académie des Sciences 52 (1861) 1094-1104.

[7] R. Bricard, Mouvement d'un solide dont tous les points décrivent des lignes sphériques, Comptes Rendus des Séances de l'Académie des Sciences 123 (1896) 39.

[8] R. Bricard, Mémoire sur la théorie de l'octaèdre articulé, J. de Mathématiques pures et appliquées 3 (1897) 113-148.

[9] E. Duporcq, Sur la correspondance quadratique et rationnelle de deux figures planes et sur un déplacement remarquable, Comptes Rendus des Séances de l'Académie des Sciences 126 (1898) 1405-1406.

[10]A. Karger, Parallel manipulators and Borel-Bricard's problem, Comput. Aided Geom. Des. 27:8 (2010) 669-680.

[11]E. Duporcq, Sur le déplacement le plus général d'une droite dont tous les points décrivent des trajectoires sphériques, J. de Mathématiques pures et appliquées 5 (1898) 121-136.

[12]A. Mannheim, Principes et Développements de Géometrié cinématique, Gauthier-Villars, Paris, 1894.

[13] G. Kœnigs, Leçons de Cinématique, Librairie Scientifique A. Hermann, Paris, 1897.

[14]J. Krames, Zur Bricardschen Bewegung, deren sämtliche Bahnkurven auf Kugeln liegen (Über symmetrische Schrotungen II), Mh. Math. Phys. 45 (1937) 407-417.

[15]J. Krames, Die Borel-Bricard-Bewegung mit punktweise gekoppelten orthogonalen Hyperboloiden (Über symmetrische Schrotungen VI), Mh. Math. Phys. 46 (1937) 172-195.

[16]J.M. Selig, M. Husty, Half-turns and line symmetric motions, Mech. Mach. Theory 46:2 (2011) 156-167.

[17] O. Ma, J. Angeles, Architecture Singularities of Parallel Manipulators, Int. J. Robot. Automat. 7:1 (1992) 23-29.

[18]A. Karger, Architecture singular planar parallel manipulators, Mech. Mach. Theory 38:11 (2003) 1149-1164.

[19] G. Nawratil, On the degenerated cases of architecturally singular planar parallel manipulators, J. Geom. Graphics 12:2 (2008) 141-149. 
[20] O. Röschel, S. Mick, Characterisation of architecturally shaky platforms, in: J. Lenarcic, M. Husty (Eds.), Advances in Robot Kinematics: Analysis and Control, Kluwer, 1998, pp. 465474.

[21] K. Wohlhart, From higher degrees of shakiness to mobility, Mech. Mach. Theory 45:3 (2010) 467-476.

[22] A. Karger, Architecturally singular non-planar parallel manipulators, Mech. Mach. Theory 43:3 (2008) 335-346.

[23]G. Nawratil, A new approach to the classification of architecturally singular parallel manipulators, in: A. Kecskemethy, A. Müller (Eds.), Computational Kinematics, Springer, pp. 349-358.

[24]M. Husty, A. Karger, Self-motions of Griffis-Duffy type platforms, Proceedings of IEEE conference on Robotics and Automation, San Francisco, USA, 2000, pp. 7-12.

[25] M. Husty, A. Karger, Architecture singular parallel manipulators and their self-motions, in: J. Lenarcic, M.M. Stanisic (Eds.), Advances in Robot Kinematics, Kluwer, 2000, pp. 355-364.

[26]X. Kong, C. Gosselin, Generation of Architecturally Singular 6-SPS Parallel Manipulators with Linearly Related Planar Platforms, Electronic Journal of Computational Kinematics 1:1 (2002) 9 pages.

[27] A.J. Sommese, C.W. Wampler, Numerical solution of systems of polynomials arising in engineering and science, World Scientific Publishing, Singapore, 2005.

[28] M. Husty, P. Zsombor-Murray, A Special Type of Singular Stewart Gough platform, in: J. Lenarcic, B. Ravani (Eds.), Advances in Robot Kinematics and Computational Geometry, Kluwer, 1994, pp. 439-449.

[29] K. Wohlhart, Architectural Shakiness or Architectural Mobility of Platforms, in: J. Lenarcic, M.M. Stanisic (Eds.), Advances in Robot Kinematics, Kluwer, 2000, pp. 365-374.

[30]P. Zsombor-Murray, H. Husty, D. Hartmann, Singular Stewart-Gough Platforms with Spherocylindrical and Spheroconical Hip Joint Trajectories, Proceedings of 9th IFToMM World Congress on the Theory of Machines and Mechanisms, Milano, Italy, 1995, pp. 1886-1890.

[31]M. Husty, A. Karger, Self motions of Stewart-Gough platforms: an overview, Proceedings of the workshop on fundamental issues and future research directions for parallel mechanisms and manipulators, Quebec City, Canada, 2002, pp. 131-141.

[32]A. Karger, Singularities and self-motions of a special type of platforms, in: J. Lenarcic, F. Thomas (Eds.), Advances in Robot Kinematics: Theory and Applications, Springer, 2002, pp. 155-164.

[33]A. Karger, Singularities and self-motions of equiform platforms, Mech. Mach. Theory 36:7 (2001) 801-815.

[34]A. Karger, Parallel Manipulators with Simple Geometrical Structure, in: M. Ceccarelli (Ed.), Proceedings of the 2nd European Conference on Mechanism Science, Springer, 2008, pp. 463470.

[35] G. Nawratil, Self-motion of TSSM manipulators with two parallel rotary axes, ASME J. Mech. Robot. 3:3 (2011) 031007.

[36] G. Nawratil, Self-motions of parallel manipulators associated with flexible octahedra, in: M. Hofbaur, M. Husty (Eds.), Proceedings of the Austrian Robotics Workshop, Hall in Tyrol, Austria, 2011, pp. 232-248. 
[37] G. Nawratil, Flexible octahedra in the projective extension of the Euclidean 3-space, J. Geom. Graphics 14:2 (2010) 147-169.

[38] G. Nawratil, A remarkable set of Schönflies-singular planar Stewart Gough platforms, Comput. Aided Geom. Des. 27:7 (2010) 503-513.

[39]A. Karger, M. Husty, Classification of all self-motions of the original Stewart-Gough platform, Comput. Aided Des. 30:3 (1998) 205-215.

[40]A. Karger, New Self-Motions of Parallel Manipulators, in: J. Lenarcic, P. Wenger (Eds.), Advances in Robot Kinematics: Analysis and Design, Springer, 2008, pp. 275-282.

[41] A. Karger, Self-motions of Stewart-Gough platforms, Comput. Aided Geom. Des. 25:9 (2008) $775-783$.

[42]F. Geiß, F.-O. Schreyer, A family of exceptional Stewart-Gough mechanisms of genus 7, in: D.J. Bates, G. Besana, S. Di Rocco, C.W. Wampler (Eds.), Interactions of Classical and Numerical Algebraic Geometry, Contemporary Mathematics 496, American Mathematical Society, 2009, pp. 221-234.

[43] G. Nawratil, Types of self-motions of planar Stewart Gough platforms: submitted to Meccanica.

[44] G. Nawratil, Basic result on type II DM self-motions of planar Stewart Gough platforms, in: E.Ch. Lovasz, B. Corves (Eds.), Mechanisms, Transmissions and Applications, Springer, 2011, pp. 235-244.

[45]G. Nawratil, Necessary conditions for type II DM self-motions of planar Stewart Gough platform: submitted to special issue "Recent Advances in Applied Geometry" of Comput. Aided Geom. Des.

[46]G. Nawratil, Stewart Gough platforms with a type II DM self-motion: submitted to J. Geometry.

[47]S. Mielczarek, M. Husty, M. Hiller, Designing a redundant Stewart-Gough platform with a maximal forward kinematics solution set, Proceedings of the International Symposium of Multibody Simulation and Mechatronics, Mexico City, Mexico, 2002, 12 pages.

[48]J. Borras, F. Thomas, C. Torras, Singularity-invariant leg rearrangements in doubly-planar Stewart Gough platforms, Proceedings of Robotics Science and Systems, Zaragoza, Spain, 2010, 8 pages.

[49] O. Bottema, B. Roth, Theoretical Kinematics, Series in applied mathematics and mechanics Vol. 24, North-Holland Publishing Company, 1979.

[50]M.G. Darboux, Sur les déplacements d'une figure invariable, Comptes Rendus des Séances de l'Académie des Sciences 92 (1881) 118-121.

[51]A. Mannheim, Etude d'un déplacement particulier d'une figure de forme invariable, Rendic. Circ. Math. Palermo 3 (1889) 131-144.

[52]G. Nawratil, Self-motions of planar projective Stewart Gough platforms: submitted to Advances in Robot Kinematics, Innsbruck, Austria, 2012.

[53] A. Karger, Self-motions of 6-3 Stewart-Gough type parallel manipulators, in: J. Lenarcic, M.M. Stanisic (Eds.), Advances in Robot Kinematics: Motion in Man and Machine, Springer, 2010, pp. 359-366. 